\title{
The role of copper(II) in the aggregation of human amylin
}

\author{
Alessandro Sinopoli, ${ }^{1}$ Antonio Magrì, ${ }^{2}$ Danilo Milardi, ${ }^{2}$ Matteo Pappalardo, ${ }^{3}$ Pietro Pucci ${ }^{4}$, Angela \\ Flagiello ${ }^{4}$, Jeremy J. Titman, ${ }^{5}$ Vincenzo Giuseppe Nicoletti, ${ }^{6,7}$ Giuseppe Caruso, ${ }^{8}$ Giuseppe \\ Pappalardo, ${ }^{2 *}$ Giuseppe Grasso ${ }^{3 *}$
}

1 Dottorato Internazionale in Biomedicina Traslazionale, Università degli Studi di Catania

2 Istituto Biostrutture e Bioimmagini, CNR, Via P. Gaifami 18, 95126, Catania, Italy

3 Dipartimento di Scienze Chimiche, Università degli Studi di Catania, Viale Andrea Doria 6, 95125, Catania, Italy.

4 Dipartimento di Scienze Chimiche, Università degli Studi di Napoli Federico II, Via Cintia 4, 80126, Napoli, Italy.

5 School of Chemistry, University of Nottingham, University Park, Nottingham NG7 2RD, U.K.

6 Dipartimento di Scienze Biomediche (Sezione di Biochimica), Università degli Studi di Catania

7 Istituto Nazionale di Biostrutture e Biosistemi (INBB) - sez. Biomolecole.

8 Dottorato Internazionale in Neurobiologia, Università degli Studi di Catania

*Correspondence to:

Giuseppe Pappalardo, Istituto Biostrutture e Bioimmagini, CNR, Viale P. Gaifami 18, 95126, Catania, Italy,

e-mail: giuseppe.pappalardo@enr.it

Giuseppe Grasso, Dipartimento di Scienze Chimiche, Università di Catania, Viale Andrea Doria 6, 95125, Catania, Italy. e-mail: grassog@unict.it 
Number of text pages: 31 Number of figures: $15 \quad$ Number of tables: 2 


\section{Abstract}

Amylin is the 37-residue peptide hormone produced by the islet $\beta$-cells in the pancreas and the formation of amylin aggregates is strongly associated with $\beta$-cells degeneration in type 2 diabetes, as demonstrated by more than $95 \%$ of patients exhibiting amylin amyloid upon autopsy.

It is widely recognized that metal ions such as copper(II) have been implicated in the aggregation process of amyloidogenic peptides such as $\mathrm{A} \beta$ and $\alpha$-synuclein and there is evidence that also amylin self-assembly is largely affected by copper(II). For this reason, in this work, the role of copper(II) in the aggregation of amylin has been investigated by several different experimental approaches. Mass spectrometric investigations show that copper(II) induces significant changes in the amylin structure which decrease the protein fibrillogenesis as observed by ThT measurements. Accordingly, solid-state NMR experiments together with computational analysis carried out on a model amylin fragment confirmed the non fibrillogenic nature of the copper(II) induced aggregated structure. Finally, the presence of copper(II) is also shown to have a major influence on amylin proneness to be degraded by proteases and cytotoxicity studies on different cell cultures are reported. 


\section{Key Words}

IAPP, Mass Spectrometry, Diabetes, neurotoxicity, copper

\section{Introduction}

Conformational disease is a general term often used to identify a number of disorders which are characterized by aggregation and deposition of specific proteins. Although the physiological roles of some of these aggregation-prone proteins have yet to be fully elucidated, ${ }^{1}$ it is possible to distinguish several different pathologies depending on the aggregation of a specific protein, i.e. $\beta$-amyloid for Alzheimer's Disease, ${ }^{2} \alpha$-synuclein for Parkinson's Disease, etc. In these diseases, the proteins in question are known to convert from their native functional state into highly organized fibrillar aggregates. In the case of amylin, ${ }^{3,4}$ the observation of amyloid deposits in islets of Langerhans of individuals with type 2 diabetes was for long time considered not important, probably because while researchers studying other pathologies were looking for protein to blame that could be responsible for the possible pathogenic mechanism, the research field of type 2 diabetes was already well established and mainly directed towards the study of insulin resistance. ${ }^{5}$ Many researchers regarded the observed failure of beta-cells only as a secondary event due to some elusive mechanism of "glucose toxicity". In addition, it is important to note that amylin in mice or rats have a different amino acid sequence which confer to the amylin molecules different aggregation properties. ${ }^{6}$ Therefore, the use of these animals as models hindered the recognition of the connection between amylin aggregation and the pathology.

In recent years, it has been documented that aggregates of amylin are directly toxic to beta-cells ${ }^{7}$ and much effort has been put toward the investigation of the possible factors triggering such aggregation. ${ }^{8-}$

${ }^{10}$ Indeed, a common feature of all amyloid deposits is the association in situ with a number of cofactors such as membrane lipids, other proteins, glycosaminoglycans and metals. ${ }^{11}$ In the case of 
amylin, many modulators of its aggregating properties have been identified. ${ }^{12-21}$ In this scenario, the role that some metal ions have in the aggregation of human ${ }^{22-24}$ or rat $^{25}$ amylin and its fragments has also been investigated in recent years. While zinc(II) ions have been shown to inhibit the formation of aggregated and toxic forms of human amylin, ${ }^{22}$ providing a possible mechanism between the zinc transporter ZnT8 and type II diabetes, ${ }^{26}$ the role of copper(II) ions in the aggregation and dyshomeostasis of amylin has been controversial. ${ }^{27}$ Indeed, while previous studies correlated copper(II) ability to inhibit amylin fibrillation with toxicity, ${ }^{23,28}$ others pointed out that copper(II) may contribute to cytotoxicity by inducing amylin to form oligomers, which may contribute more to cell death than fibrils do. ${ }^{24}$ Mass spectrometry has also been applied to elucidate the structure of copper(II)-amylin complexes and the putative binding sites. ${ }^{29,30}$ However, there is a lack of study regarding the structure of copper(II)-induced amylin aggregates which could provide useful information for developing therapeutic strategies that could inhibit metal-induced aggregation and maybe even reverse it. Indeed, amylin homeostasis seems to be regulated also by the action of some metalloproteases which have been found to be able to degrade this hormone. ${ }^{31-33}$ The further role that copper(II) might have in modulating the degradation of amylin by these enzymes has not been studied, even if this metal ion has been proven to be able to tune the enzymatic activity of some of these metalloproteases. ${ }^{34-36}$ The copper(II)driven conformational changes on h-amylin ought to have a large impact on its in vivo homeostasis. Particularly, as a major contribution to amylin catabolism in vivo seems to be given by its degradation by metalloproteases, ${ }^{31-33}$ we have also studied the effect of copper(II) on amylin degradation by IDE. The latter seems to be involved in the proteolytic processing of many other different substrates, ${ }^{37,38}$ which often also function as modulators of its activity ${ }^{39,40}$ and degradation of amylin by IDE has already been reported in the literature. ${ }^{41,42}$ However, the cleavage sites of IDE on amylin seem to depend on the particular conditions used, as different peptide fragments are reported in different published works. ${ }^{41,42}$ In this scenario, considering also the link that might exist between copper(II) 
dyshomeostasis and type II diabetes, ${ }^{43}$ we have investigated the role of copper(II) ions into the packing and aggregating processes of human amylin (h-amylin) by several different experimental approaches. Particularly, information on the kinetics, the structure and the proneness of amylin aggregates to be degraded by metalloproteases have been obtained and discussed. Moreover, owing to the observation that amylin receptors, being shared between amylin and beta-amyloid peptides, appear to be also involved in the pathophysiology of Alzheimer's disease ${ }^{44}$ and thus representing a molecular link between the two epidemiologically associated conditions, we have also measured the effect of copper on the toxic activity of h-amylin and its short peptide 17-29 on neuronal cell cultures.

\section{Experimental}

\section{Materials and methods}

Insulin-degrading enzyme (IDE), His-Tag, rat, recombinant, Spodoptera frugiperda was purchased from CALBIOCHEM. Subtilysin, Elastase, Chymotrypsin and $\mathrm{D}_{2} \mathrm{O}$ were purchase from Sigma, while ZipTip $_{\mathrm{C} 18}$ pipette tips were from Millipore (Billerica, MA, USA). h-amylin was purchased from BACHEM, while the 17-29 h-amylin fragment were synthesized as reported in ref. 10. For solid-state NMR measurements labelled 17-29 h-amylin fragments were synthesized: 1) Ser29-labelled with 13C3 and $15 \mathrm{~N}:[\mathrm{Rt}=20.30 \mathrm{~min}]$. Mass calculated for: $12 \mathrm{C} 5613 \mathrm{C} 3 \mathrm{H} 9214 \mathrm{~N} 17$ 15N O20 = 1376.5 ; ESI-MS [Obsd m/z: $(\mathrm{M}+\mathrm{H})^{+}$1377.8] hIAPP17-29; 2) Val17-labelled with $13 \mathrm{C} 5$ and 15N: [Rt $\left.=21.40 \mathrm{~min}\right]$. Mass calculated for: 12C54 13C5 H92 14N17 15N O20 = 1378.5; ESI-MS [Obsd m/z: $(\mathrm{M}+\mathrm{H})+$ 1379.7]. The solvent gradients needed for labelled peptides purification were the same used for nonlabelled 17-29 h-amylin fragment and are reported in ref. 10. 


\section{Simulations}

Constant Temperature Molecular Dynamics (CTMD) simulations of the self-assembling of the Nacetylated and C-amydated 17-29 h-amylin fragment were performed by using the software CHARMM33. The peptide system was modelled by explicitly considering all heavy atoms and the polar hydrogen atoms bound to nitrogen and oxygen. The CHARMM 19 potential function was adopted and default cut-offs for long range interactions were used, i.e. a shift function of $1 \AA$ was employed with a cut-off at $7.5 \AA$ for both the electrostatic and van der Waals terms. Langevin dynamics with a friction value of $0.15 \mathrm{ps}^{-1}$ were used. This friction coefficient is much smaller than the one of water $\left(43 \mathrm{ps}^{-1}\right.$ at $330 \mathrm{~K}$ computed as $3 \pi \eta \mathrm{d} / \mathrm{m}$, where $\eta$ is the viscosity of water at $330 \mathrm{~K}$, and $\mathrm{d}$ and $\mathrm{m}$ are the effective diameter, i.e., $2.8 \AA$, and mass of a water molecule, respectively) to allow for sufficient sampling within the time scale (ns) of the simulations. It has been demonstrated that the small value of friction coefficient adopted does not influence the thermodynamic properties of the system, thus ruling out the possibility of kinetic traps along the aggregation pathway. The implicit solvent model EEF1 was employed to simulate the aqueous solvent. The SHAKE algorithm was used to fix the length of the covalent bonds involving hydrogen atoms; this option allows reducing the integration time step down to a value of 2 fs without affecting the reliability of the simulations. Furthermore, the non-bonded interactions were updated every ten dynamics steps and the coordinate frames were saved every 20 ps. A 20 ns implicit water MD simulation was performed on each single peptide at $\mathrm{T}=300 \mathrm{~K}$ to equilibrate the monomer. Ten replicas of the equilibrated monomer were then used to simulate aggregation in water. In the initial positions there were neither inter- or intramolecular contacts, i.e. the ten peptides were completely unfolded and not interacting in space. All simulations were started from random positions, orientations and conformation of the peptide copies. The final assembly was simulated in a cubic box of $600 \AA$ side and re-equilibrated for $20 \mathrm{~ns}$ at $300 \mathrm{~K}$. These conditions correspond to a final peptide concentration of $10 \mu \mathrm{M}$. Additional MD runs carried out 
by varying the size of the solvent box (i.e. at different peptide concentrations) did not modify the outcome of the simulations. Next, productive MD simulations were carried out at $300 \mathrm{~K}$ for total $1 \mu \mathrm{s}$. The polar order parameter was considered to monitor the aggregation process as described elsewhere. The parameter P1, which is widely used to study the properties of anisotropic fluids, is defined as:

$$
\overline{P_{1}}=\frac{1}{N} \sum_{i=1}^{N}\left(\overrightarrow{z_{i}} \vec{d}\right)
$$

where (the director) is a unit vector defining the preferred direction of alignment, is the molecular unit vectors linking the peptide's $\mathrm{N}$ - and $\mathrm{C}$ - termini and $\mathrm{N}$ is the number of molecules in the simulation box, i.e. three in the present study. The polar P1 describes how the molecular vectors point in the same direction, and discriminates between parallel and antiparallel/mixed ordered aggregates.

The Root Mean Square Deviation (RMSD) analysis of an aggregate is usually employed to check the stability of the simulated system. The RMSD between two structures (corresponding to times ti and $\mathrm{ti}+1$ of the trajectory) is defined as follows:

$$
\operatorname{RMSD}\left(t_{i}+t_{i+1}\right)=\sqrt{\frac{1}{N} \sum_{i=1}^{N}\left|\vec{r}_{i}\left(t_{i}\right)-\vec{r}_{i+1}\left(t_{i+1}\right)\right|^{2}}
$$

where $r_{i}$ and $r_{i+1}$ represent the position vectors of the $\mathrm{N}$ protein atoms calculated at time $i$ and $i+1$ respectively.

\section{Solid-State NMR}


13C CPMAS NMR spectra were recorded at room temperature on a Varian Infinity plus spectrometer at a Larmor frequency of $75.47 \mathrm{MHz}$ using a $3.2 \mathrm{~mm}$ MAS probe spinning at $25 \mathrm{kHz}$. TPPM decoupling at a field of $100 \mathrm{kHz}$ was applied during acquisition of the $13 \mathrm{C}$ spectrum. Chemical shifts are quoted relative to TMS, using adamantane as a secondary external reference.

\section{Mass Spectrometry}

The peptide fragments generated by the enzymatic digestion of amylin by IDE were analyzed by using a Finnigan LCQ DECA XP PLUS ion trap spectrometer operating in the positive ion mode and equipped with an orthogonal ESI source (Thermo Electron Corporation, USA). Sample solutions were purified by ZIPTIPC18 and after dilution with $50 \mu \mathrm{l}$ of water and $50 \mu \mathrm{l}$ of Methanol were injected into the ion source at a flow-rate of $5 \mu \mathrm{l} / \mathrm{min}$, using nitrogen as drying gas. The mass spectrometer operated with a capillary voltage of $46 \mathrm{~V}$ and capillary temperature of $250^{\circ} \mathrm{C}$, while the spray voltage was 4.3 $\mathrm{kV}$.

\section{Hydrogen/ Deuterium exchange experiments}

The H/D exchange experiments were performed on h-amylin before and after incubation with copper (II) using a protein: metal molar ratios of 1:10. The latter condition was applied in order to ensure that the amount of free amylin in solution was negligible. Indeed, in figure 1S of the Supplementary Material the mass spectra and the peak assignment of three amylin-copper solutions at various molar ratios are reported. It can be noted that the 1:10 molar ratio ensures the maximum amount of amylincopper complex (the peak assigned to free amylin is absent in this case) without the formation of other species other than the 1:1 metal-amylin complex. For this reason this molar ratio was chosen for the $\mathrm{H} / \mathrm{D}$ exchange and the limited proteolysis (see below) experiments. The H/D exchange was carried out as follows: the protein sample $(600 \mathrm{pmol} / \mu \mathrm{l})$ was equilibrated for $15 \mathrm{~min}$ at $25^{\circ} \mathrm{C}$ in ammonium acetate 
$10 \mathrm{mM}$ pH 7. Deuterium exchange was initiated by 10-fold diluting the sample with ammonium acetate $10 \mathrm{mM}$, previously prepared using $\mathrm{D}_{2} \mathrm{O}$ at the appropriate $\mathrm{pD}$. At various exchange times (from $15 \mathrm{sec}$ to $1 \mathrm{~h}$ ), aliquots of $1.5 \mathrm{nmol}$ of protein were withdrawn and rapidly analysed by LC/MS by direct injection into a HPLC coupled to a ZQ single quadrupole instrument (Waters), using a $30 \times 0.46 \mathrm{~mm}$ reverse phase perfusion column (POROS $10 \mathrm{R} 1$, Applied Biosystems). The protein was eluted at a flow rate of $0.5 \mathrm{ml} / \mathrm{min}$ with a $30-95 \% \mathrm{CH} 3 \mathrm{CN}$ gradient in $0.1 \%$ TFA in $1 \mathrm{~min}$.

The HPLC analysis was quickly performed with cold solvents keeping the column in an ice-bath to reduce the $\mathrm{H} / \mathrm{D}$ back-exchange kinetics. In these conditions, deuterium ions from the amino acid side chains and the peptide C-terminus are rapidly substituted with hydrogens while exchange from amide bonds is much slower and can be measured. Duplicate analyses were performed for each time point.

\section{Limited Proteolysis Experiments}

The limited proteolysis experiments were performed on h-amylin before and after incubation with copper (II) using a protein: metal molar ratio of 1:10. Protein samples were individually treated with chymotrypsin, subtilisin and elastase. Enzymatic digestions were all performed in $50 \mathrm{mM}$ ammonium hydrogen carbonate $\mathrm{pH} 7$ at $25^{\circ} \mathrm{C}$ by using an $\mathrm{E}: \mathrm{S}$ ratio $(\mathrm{w} / \mathrm{w})$ of $1: 100$ in the case of chymotrypsin and 1:50 in the case of subtilisin and elastase. After $2 \mathrm{~h}$ incubation, digestion was stopped by adding trifluoroacetic acid and aliquots of the peptide mixtures were directly analysed by MALDIMS.

MALDI-MS analyses were carried out on a Voyager DE-PRO MALDI TOF (ABI Sciex) mass spectrometer equipped with a reflectron analyzer and used in delayed extraction mode with Voyager control software. $1 \mu \mathrm{l}$ of peptide mixture was mixed with an equal volume of a-cyano-4hydroxycynnamic acid as matrix $(10 \mathrm{mg} / \mathrm{ml}$ in $0.2 \% \mathrm{TFA}$ in $70 \%$ acetonitrile $)$, loaded onto the metallic sample plate and air dried. Mass calibration was performed using the internal standard calibrants added 
to the matrix. MALDI-MS data were acquired over a mass range of $250-6000 \mathrm{~m} / \mathrm{z}$ in the positive mode using the software provided by the manufacturer.

\section{Sample preparation}

All peptide samples were subjected to a disaggregating procedure to remove any existing preaggregated form before carrying out each experiment. The protocol consists in dissolving samples in 1,1,1,3,3,3-hexa-fluoro-2-propanol (HFIP) at a concentration of $1 \mathrm{mg} / 1 \mathrm{ml}$ and incubating them at $37^{\circ} \mathrm{C}$ for $1 \mathrm{~h}$. HFIP is then removed by gentle streaming with argon and the resulting peptide film dissolved again in $1 \mathrm{ml} \mathrm{HFIP,} \mathrm{frozen} \mathrm{at}-30{ }^{\circ} \mathrm{C}$ for $4-5$ hours and lyophilized overnight. The lyophilized samples were then solubilized in MilliQ water to a concentration of $2 \times 10-5 \mathrm{M}$ adjusting the $\mathrm{pH}$ value just before the CD or ThT measurements.

\section{Circular Dichroism spectroscopy}

The Circular Dichroism (CD) spectra were obtained at $37{ }^{\circ} \mathrm{C}$ under a constant flow of $\mathrm{N}_{2}$ on a Jasco J810 spectropolarimeter equipped with a Peltier thermoelectric type temperature control system. Experimental measurements were conducted at $\mathrm{pH} 7$ using $1 \mathrm{~cm}$ or $0.1 \mathrm{~cm}$ path length cuvettes. The CD spectra were recorded in the UV region (190-260 nm) at peptide concentration of $2 \times 10^{-5} \mathrm{M}$ in MilliQ water. The CD spectra were acquired every 20 min over an experimental time course of 1500 min. CD intensities are expressed as mean residue ellipticity $[\theta]\left(\mathrm{deg} \mathrm{cm}^{2} \mathrm{dmol}^{-1}\right)$.

\section{Thioflavin-T assay}

Fluorescence emission spectra of Thioflavin-T (ThT) undergo a red shift upon incorporation into $\beta$ sheet amyloid structures. Fluorescence was monitored as a function of time in a 96-well plate using a VARIOSKAN plate reader. The measurements were carried out using, as a control, the time 
dependence of the fluorescence of ThT solutions without the peptide. Samples were monitored over a $1400 \mathrm{~min}$ period at an excitation wavelength of $450 \mathrm{~nm}$ and the emission was $480 \mathrm{~nm}$.

\section{Cell cultures and h-amylin cytotoxicity}

Human neuroblastoma cell line (SH-SY5Y: ATCC® CRL-2266 ${ }^{\mathrm{TM}}$ ) and insulinoma cell line (RIN-m: ATCC $($ CRL-2057TM) were cultured in DMEM/F12 medium (1:1) and RPMI 1640 respectively, supplemented with 10\% (v/v) fetal bovine serum (FBS), penicillin (50 U/ml) and streptomycin $(50$ $\mu \mathrm{g} / \mathrm{ml})$. The cells were maintained in a humidified environment at $37^{\circ} \mathrm{C}$ and $5 \% \mathrm{CO}_{2}$ and cultured in 75 $\mathrm{cm}^{2}$ culture flasks. The medium was replaced twice a week and cells were splitted upon reaching about $80 \%$ confluence. The day prior to treatment cells were harvested and seeded in 48 -well plates at a density of 25000 cells/well. The cells were treated as soon as they reached maximal confluence.

To evaluate the cytotoxicity produced by the h-amylin peptide, insulinoma and neuroblastoma cell cultures were treated for 48 hours with different preparations of 17-29 h-amylin peptide and full length protein, and viability was then measured by the classic MTT protocol.

\section{MTT ASSAY}

The toxicity of the peptides was measured through the determination of cell viability of treated cells compared to control (untreated cells), by MTT [3-(4,5-dimethylthiazol-2-yl)-2,5-diphenyltetrazolium bromide] assay. The test is based on the ability of the mitochondrial enzyme, cytochrome c oxidase and succinate dehydrogenase, to reduce yellow tetrazole into purple formazan that, being unable to cross the plasma membrane, will accumulate within the living cells. Solubilization of formazan crystals with an appropriate detergent will produce a purple solution, and absorbance measureswill indicate cells viability. After treatment with the peptide preparations, cell cultures were incubated for 1 hour at $37^{\circ} \mathrm{C}$ 
in the MTT solution $(1 \mathrm{mg} / \mathrm{ml}$ in PBS); the formed crystals were melted with DMSO. After solubilization of the formazan crystals a microplate reader was used to read the absorbance at 590nm.

\section{Results and discussion}

To study the conformational changes that copper(II) ions could induce on h-amylin, solutions of the latter $(20 \mu \mathrm{M})$ were diluted 10 -fold by the addition of the appropriate $\mathrm{D}_{2} \mathrm{O}$ amount and deuterium incorporation was monitored by sampling the incubation mixture at different interval times followed by cold acid quenching and fast LC/MS analysis. Since H/D exchange of amide deuterons occurs very slowly, the increase in molecular mass of the protein sample constituted a direct measurement of deuterium incorporation at peptide amide linkages. The number of amide protons exchanged with deuterium in the native form of h-amylin and h-amylin-Cu (II) complex as a function of time is reported in Figure 1. Native h-amylin showed two distributions of multiply charged ions in the ES mass spectra, eventually originating two curves, thus suggesting the occurrence of two different conformations of the protein having a clear difference in the exchange kinetics. Following 60 min of reaction, the two protein conformations exchanged a total of 25 and 5 hydrogens respectively, thus indicating the existence of an equilibrium between a higher unstructured conformation and a more compact structure. The analysis of the relative abundances of the two conformers as estimated by mass peak intensities showed that h-amylin in solution essentially adopts an unstructured conformation with a minor fraction of molecules assuming a more compact structure. Following $\mathrm{Cu}$-complex formation, we observed the occurrence of a single compact conformation of h-amylin that exchanged very few protons. This behaviour almost resembled that of the most stable conformation observed in the analysis of the isolated protein. A possible interpretation of the data suggests that in solution h-amylin gives rise to an equilibrium between two conformations, with the more unstructured state being the most 
populated. The presence of copper(II) greatly shifted the equilibrium towards the more compact structure that eventually constitutes the only existing conformer.

A further confirmation of the copper(II)-induced change in the conformation was obtained by carrying out limited proteolysis experiments using an array of different enzymes including Subtilysin, Elastase and Chymotrypsin in enzyme/substrate ratios of 1/50 for Subtilysin and Elastase or 1/100 in the case of Chymotrypsin (see Figure $2 \mathrm{~S}$ of the supplementary material). Limited proteolysis was carried out at $37^{\circ} \mathrm{C}$ in $50 \mathrm{mM}$ of ammonium hydrogen carbonate hydrolysis buffer at $\mathrm{pH} 7$ for $2 \mathrm{~h}$. The MALDI-MS analyses allowed to monitor the changes in the cleavage sites after copper(II) addition (see Figure 2). It was so possible to establish that the copper(II) binding site is located in the region containing the His 18 residue which is likely the metal binding site. This result was further confirmed by using IDE as amylin proteolytic enzyme (see introduction). Figure 3 shows that, in the presence of copper(II), the cleavage sites of IDE on amylin (determined by the MS detection of the IDE-produced amylin fragments, data not shown) decreased in number but also, more importantly, changed drastically. It was particularly interesting that, apart from the expected inhibitory role of copper(II) on amylin degradation by IDE (data not shown), ${ }^{34,36,45}$ the enzyme was no longer able to cut the peptide in the middle part of its sequence, indicating that conformational changes driven by copper(II) binding to this segment of peptide chain renders the latter unavailable for enzymatic hydrolysis.

Once it was assessed that copper(II) induces a conformational change on the h-amylin molecules, we decided to investigate the aggregation kinetics of h-amylin in the presence and in the absence of the metal ion. For this purpose, $\mathrm{CD}$ as well as ThT fluorescence measurements were carried out on the various samples. In Figure 4 the $\mathrm{CD}$ spectra of h-amylin incubated at $37^{\circ} \mathrm{C}$ with copper (II) at different times are reported together with the $\mathrm{CD}$ profile of the protein alone. In the absence of copper the polypeptide adopts a random coil conformation, while in the presence of metal ions h-amylin conformation shifts toward a mix of random coil and $\alpha$-helix structures. Indeed, the minimum near 200 
$\mathrm{nm}$ undergoes a red shift over time which indicates a stabilization of an $\alpha$-helix conformation, while there is not the presence of the negative peak at $216 \mathrm{~nm}$ over time, confirming the absence of $\beta$-sheet structures. $^{46}$ Therefore, although copper induces a conformational change onto the h-amylin molecule which shifts the equilibrium toward a more structured form (see MS results), the lack of $\beta$-sheet structures as seen by CD indicates the absence of an induced fibrillar form of the protein. This result was further confirmed by ThT fluorescence measurements. Figure 5 shows the h-amylin ThT kinetics, recorded at $\mathrm{pH} 7.0$ in the absence and in the presence of copper(II) (1:1 ratio). In the absence of copper(II) a short lag phase (near 100 min.) followed by a rapid growth of ThT fluorescence was observed. In contrast, in the presence of copper the lag phase was almost 3 times longer and the fluorescence intensity after 600 minutes was more than 2 times lower, confirming the absence of metal induced fibrillar form of h-amylin. Indeed, copper(II) induces a conformational change on the protein that, although is in a more structured state (H/D exchange MS and CD results), is less prone to aggregation, in accordance with previous works. ${ }^{23,46}$

Finally, in order to get a closer insight onto the mechanism by which copper(II) induces the above reported changes on both the conformation and aggregation properties of h-amylin, we have used the 17-29 h-amylin fragment (Figure 3) in order to carry out simulations as well as solid-state NMR measurements on the aggregated state. The reason and the validity of using this particular peptide portion has already been reported elsewhere. ${ }^{10}$ Figure 6 shows 13C CPMAS spectra of three 17-29 hamylin fragments (A-C). Sample A is the native 17-29 h-amylin fragment, with U-13C3-15N1-Ser29 isotopic labelling. Sample B is the same fragment, which has been bound to copper(II) ions in a 1:1 ratio. Sample C is 17-29 h-amylin fragment with $\mathrm{U}-13 \mathrm{C} 5-15 \mathrm{~N} 1 \mathrm{Val17}$ which has also been bound to copper(II) ions in a 1:1 ratio. Observed shifts are recorded in ppm (see Table 1), spectra were recorded at $25 \mathrm{kHz}$. All spectra displayed a broad peak arising from natural abundance $13 \mathrm{C}$ nuclei which are present in all residues. Sample A showed three sharp resonances for the carbonyl, $\alpha$ and $\beta$ carbons of 
the universally labelled Ser29 residue consistent with the values reported in the literature for full length h-amylin. ${ }^{47}$ Sample B clearly displayed shift differences compared to A, consistent with a hyperfine coupling to the paramagnetic copper(II) ions present. Only two resonances were observed, which were attributed to the $\alpha$ and $\beta$ carbons. This suggests that the carbonyl signal has been broadened due to paramagnetic relaxation effects. Sample $\mathrm{C}$ also displayed large differences form the expected shifts due to paramagnetic couplings. These data indicate that both Val17 and Ser29 residues at each end of the fragment experience significant paramagnetic shifts and are therefore both close in space to the copper(II) ion.

Far-Uv CD spectroscopy was used also to monitor the h-amylin 17-29 peptide conformational changes associated with fibril formation. Aggregation kinetics monitored by CD spectroscopy were measured in the presence of copper(II) at $\mathrm{pH} 7$ in water as above described, showing that copper(II) modifies the polypeptide conformation. The CD profile shown in Figure 7 appears to be a mixture between random coil and folded structure. The spectra recorded without metal showed a typical random coil conformation but after copper(II) addition, the $222 \mathrm{~nm}$ ellipticity increased while the $200 \mathrm{~nm}$ ellipticity was reduced. It is important to note that the CD spectra relevant to h-amylin 1-37 and the shorter peptide h-amylin 17-29 appear quite different. The analysis of the CD profiles suggests that copper(II) coordination brings structuring effects into the polypeptide backbone. This should occur particularly in the peptide region involved in metal ion binding where the coordinated amino acid residues induce the adoption of a peptide bent conformation that is mainly driven by the geometrical coordination preferences of the metal ion and by the stability of the formed chelate rings. This new locally defined conformation contributes with a distinctive dichroism to the observed spectral pattern. Such an effect becomes more evident in the $\mathrm{CD}$ curves of the shorter h-amylin 17-29 peptide sequence. The difference spectra obtained by subtracting the $\mathrm{CD}$ traces recorded without metal from those obtained in the presence of copper(II), should, in principle, give an indication of the difference of backbone 
conformation caused by copper(II) complexation. Indeed, what we observe is that the resultant CD curves give strikingly similar CD profiles with a positive ellipticity below $200 \mathrm{~nm}$ and a negative signal centred around 217-220 nm (see Figure $3 \mathrm{~S}$ of the supplementary material). This in our opinion strongly suggests, that in both cases, copper(II) experiences a similar coordination environment and this can justify the use of the shorter peptide fragment to study the metal binding site of the full-length protein. Finally, CTMD simulations performed at $\mathrm{T}=300 \mathrm{~K}$ in implicit water have shown that the ten replicas of the 17-29 h-amylin fragment exhibited low values of both RMSD and its fluctuations (Figure 8) thus suggesting that they rapidly collapse to give a stable, compact structure.

An analysis of the secondary structure percentages averaged along the entire simulation provided information about the conformational preferences of the peptide in the condensed state (Table 2) that was found in agreement with the solid-state NMR results. Indeed, the purpose of MD simulations is to support an head-to-tail topological arrangement of 17-29 h-amylin aggregates. This information is important to support the interpretation of the solid-state NMR experiments. Actually, Val17 and Ser29 residues at each end of the 17-29 h-amylin fragment experience significant paramagnetic shifts and are therefore both close in space to the copper(II) ion. However, the C-terminus of 17-29 h-amylin is not supposed to bind copper(II) and, as a consequence, the NMR results may be explained $i$ ) assuming that copper(II) binds the N-terminus (Val17) of the peptide and induce the molecule to bend and adopt a Ushaped structure or ii) supposing an head-to-tail arrangement of the peptide aggregates. In general, little is known about the metal-binding sites in the aggregated state of peptides and it is difficult to establish by experiments which of these two hypotheses holds true when the peptide aggregates. Therefore, we resorted to CTMD simulations which pointed clearly to an head-to-tail topological arrangement of the aggregated peptides. All these simulations were performed in the absence of copper(II) ions. We are aware that MD simulations performed in the presence of copper ions would allow a better comparison with NMR results. However, the presence of a metal ion in the system would make the use of QM 
simulations much safer, as they embody charge polarization and screening, rather than the use of the purely classical MD adopted in the present work. But an obvious limitation to the use of QM simulations is the foreseeable very short length of the computed trajectory (few picoseconds for systems of the size one is interested in here by using commonly-available hardware resources) which certainly cannot fully account for the complicated peptide aggregation dynamics.

The time evolution of the secondary structure for each replica of the 17-29 h-amylin fragment, reported in Figure 9, underlines the high tendency to self-assembly in beta-sheet for this system. This observation is consistent with the RMSD curves of the 17-29 h-amylin fragment in water that are typical of a structured system with high rigidity and compact packing. Figure 10 shows a representative snapshots of the 17-29 h-amylin fragment aggregated in water. In principle, in the early steps of aggregation, all amyloidogenic peptides assembled into highly ordered $\beta$-sheet structures. During the assembly, the peptides tend to align adopting an extended $\beta$-strand conformation and a remarkable change in the local orientational order occurs. The aggregation of amyloid forming peptides may then be interpreted as an order transition and orientational order parameters are suitable to monitor the time evolution of the process. Figure 11 reports the projections along the polar order parameter P1 of the 1729 h-amylin fragment assemblies calculated as reported in the experimental section. The CTMD profiles along $\mathrm{P} 1$ show that at room temperature $\mathrm{T}=300 \mathrm{~K}$ macrostates arranged in an anti-parallel $\beta$ sheet configuration are highly populated $(0<\mathrm{P} 1<0.3)$. To a lower extent, mixed parallel/antiparallel arrangements are populated $(0.3<\mathrm{P} 1<0.7)$. At higher $\mathrm{P} 1$ values $(\mathrm{P} 1>0.7)$ where only parallel arrangements are supposed to exist, the CTMD profiles are close to zero suggesting that parallel arrangements are not likely to be present during the simulations.

All the observed conformational changes induced by incubation of peptide alone or in the presence of $\mathrm{Cu}(\mathrm{II})$, should affect not only the status of aggregation, but also its toxic property. We therefore evaluated the relationship between aggregating conditions and toxicity on insulinoma cell line culture 
and, in order to provide further proofs on the correlations between diabetes and Alzheimer's disease, we also used the neuronal cell line, SH-SY5Y, as cell culture model. Cells at full confluence were treated for 48 hours with 17-29 h-amylin preparations $(25 \mu \mathrm{M})$, that consist in pre-incubation in vitro alone or in the presence of $\mathrm{CuSO}_{4}$ (at 1:1 molar ratio) for 48 hours in PBS $0,01 \mathrm{M}, \mathrm{pH}$ 7,4. The data obtained showed a reduced viability (decreased ability to reduce MTT), compared to control (Fig. 12), in all the samples treated with 17-29 h-amylin. It cannot be excluded any further changes in the status of aggregation during the $48 \mathrm{hs}$ of cell culture treatment. This could explain the toxicity exerted by the freshly prepared (not pre-incubated) peptide (-20\%). The peptide pre-incubation alone or in the presence of $\mathrm{CuSO}_{4}$ (at 1:1 molar ratio) further increases its toxicity $(-40 \%$ and $-50 \%$ of viability respectively). The stronger decrease in cell viability observed after treatment with the peptide/Cu(II) is in accordance with the ability of copper to counteract $\beta$-sheet conformational changes and fibrillogenesis, thus prolonging the presence of more toxic and self-assembling oligomeric species. However it cannot be excluded that cell viability could also be affected by the formation of toxic free radical species produced in the presence of $\mathrm{Cu}(\mathrm{II})$. The treatment of neuroblastoma as well as insulinoma cell cultures with h-amylin 1-37 (full protein) preparations $(20 \mu \mathrm{M})$ for 48 hours, revealed that amylin toxic effect was, in this case, not affected by the presence of copper ions during the $48 \mathrm{~h}$ of in vitro pre-incubation (Figures 13 and 14). This result could be related and is in agreement with the measured differences of $\mathrm{CD}$ spectra and the absence of metal induced $\beta$-sheet structures (fibrillar form) of h-amylin.

\section{Conclusions}

Several different experimental techniques as well as CTMD simulations have been applied in order to assess the conformation of h-amylin in the presence and in the absence of copper(II). The latter metal ion has been shown to drastically affect both the fibers structure and aggregation kinetics of h-amylin, 
as well as its proneness to be degraded by proteases. Particularly, MS data indicate that h-amylin gives rise to an equilibrium between two conformations, with the more flexible state being the most populated, while CD spectra of h-amylin are in accordance with a random coil conformation and incubation of the peptide alone leads to aggregation. The presence of copper(II) induces the formation of a more compact structure that eventually constitutes the only existing conformer. However, no clear signs of $\beta$-sheet conformation could be observed from the spectroscopic patterns recorded in the presence of copper(II), thereby suggesting that the metal ion can inhibit h-amylin fibril formation. The copper(II)-h-amylin species are less prone to enzyme and metalloproteases degradation, revealing that the metal binding has to occur within the 17-29 h-amylin region and the coordination features of this peptide fragment are in progress. Solid-state NMR as well as CTMD simulations indicated the presence of mixed parallel/antiparallel arrangements, confirming the randomness of this specific metal ioninduced aggregation process.

These conformational changes can also explain the highest level of toxicity that we observed after cell culture treatment with 17-29 h-amylin fragment preincubated in the presence of $\mathrm{Cu}(\mathrm{II})$ and the absence of effects of copper on h-amylin 1-37 spontaneous evolution towards toxic species.

Finally, we believe that, as copper(II) dyshomeostasis seems to be involved in the development of type II diabetes, the reported differences observed both in the kinetics as well as in the kind of the aggregates of h-amylin in the presence and in the absence of copper(II) may have important implications in vivo which should be further investigated.

\section{Acknowledgement}

We thank FIRB "RINAME” RBAP114AMK, FIRB "Rete Nazionale per lo studio della Proteomica Umana (Italian Human ProteomeNet)" RBRN07BMCT, PRIN 2008R23Z7K and FIRB-Merit RBNE08HWLZ for partial financial support. The authors wish to thank Ms. Daniela Gemma Cartia 
who assisted

in

the

preparation

of

the manuscript. 


\section{Tables}

\begin{tabular}{||c|c||}
\hline Literature & h-amylin / ppm \\
\hline Val17 & 171.7 \\
& 58.8 \\
& 34.2 \\
& 19.6 \\
\hline Ser29 & 171.9 \\
& 64.8 \\
& 54.9 \\
\hline \hline Sample & $\mathbf{1 7 - 2 9}$ h-amylin \\
& ppm \\
\hline \hline C: Val17 & 125 \\
& 85 \\
& 20 \\
\hline B: Ser29 & 175 \\
& 85 \\
\hline & $\mathbf{1 7 - 2 9}$ h-amylin \\
& fragment/ ppm \\
\hline A: Ser29 & 175 \\
& 60 \\
& 50 \\
\hline \hline
\end{tabular}

Table1. Chemical shifts observed for 17-29 h-amylin fragments A-C. Top: reported shifts of Val17 and Ser29 in the full-length h-amylin. Bottom: observed shifts for 17-29 h-amylin fragments A-C.

\begin{tabular}{|l|l|l|l|}
\hline helix & sheet & coil & turn \\
\hline $\mathbf{0 . 0 4}$ & $\mathbf{2 7 . 7 2}$ & $\mathbf{3 8 . 0 8}$ & $\mathbf{3 4 . 1 4}$ \\
\hline
\end{tabular}

Table 2. Secondary structure averaged along simulation in water. 


\section{Figure Legends}

Figure 1: Number of amide protons exchanged with deuterium in the native form of h-amylin and hamylin-Cu (II) complex as a function of time. H-amylin showed two distributions of multiply charged ions in the ES mass spectra, eventually originating two curves indicated 1A and 1B. Discussion is in the text.

Figure 2. Main limited proteolytic sites in h-amylin (black blocks) and the $\mathrm{Cu}(\mathrm{II})$-h-amylin complex (grey blocks). Chymotrypsin digestion sites: F15, L16, F23 and L27; Elastase digestion sites: A13, S20, L27 and V32; Subtylisin digestion sites: L12, L16, L27, G33 and N35.

Figure 3: Cleavage sites of IDE on h-amylin as reported in ref. 41 (black solid arrows), as reported in ref. 42 (grey solid arrows), as obtained in this work after $2 \mathrm{~h}$ at $37^{\circ} \mathrm{C}$ of h-amylin solution $(50 \mu \mathrm{M}$ in PBS), IDE $0.2 \mu \mathrm{M}$, without (black dashed arrows) and with (grey dashed arrows) copper(II). Grey amino acidic residues refer to the amylin portion used as a model for solid-state NMR and computational studies.

Figure 4: $\mathrm{CD}$ spectra of h-amylin $2 * 10^{-5} \mathrm{M}$ in water recorded at different times of incubation at $37^{\circ} \mathrm{C}$ with equimolar copper (II). The dotted curve refers to the CD profile in the absence of the metal ion at the same experimental conditions.

Figure 5: Kinetics of fibril formation monitored by ThT fluorescence of h-amylin $\left(2 * 10^{-5} \mathrm{M}\right)$ in water solution at $\mathrm{pH}=7$ and $37^{\circ} \mathrm{C}$ without (solid line) and with (dotted line) copper(II) $\left(2 * 10^{-5} \mathrm{M}\right)$. 
Figure 6: 13C CPMAS spectra of the 17-29 h-amylin fragments. C: prepared using U-13C5-15N1 Val17 and $\mathrm{Cu}(\mathrm{II}), \mathrm{B}$ : prepared using U-13C3-15N1 Ser29 and $\mathrm{Cu}(\mathrm{II}), \mathrm{A}$ : prepared using U-13C3-15N1 Ser29 only.

Figure 7: CD spectra of 17-29 h-amylin fragment $\left(2 * 10^{-5} \mathrm{M}\right)$ recorded at different time intervals (from 0 to $21 \mathrm{~h}$ ) at $\mathrm{pH} 7.0$ and at $37^{\circ} \mathrm{C}$ in water solution in the presence of equimolar copper(II). The dotted curve refers to the $\mathrm{CD}$ profile in the absence of the metal ion at the same experimental conditions.

Figure 8: RMSD of the ten-meric assembly of the 17-29 h-amylin fragment, in water.

Figure 9: Time evolution of the secondary structure of the 17-29 h-amylin fragment during the early stages of aggregation (the first $4 \mathrm{ns)}$ in water as obtained from the analysis of hydrogen bonds in the peptide main chain by the VMD software. Here, the vertical coordinate represents the residue number from the first to the 10th replica which is plotted against time. The secondary structure is color-coded.

Figure 10: A snapshot of the ten-meric aggregate of the 17-29 h-amylin fragment in water.

Figure 11: Percentage of populations of ten-meric 17-29 h-amylin fragment assemblies plotted along the polar P1, order parameter. Schematic representations of the aggregates (black arrows) are depicted to show that order parameter P1 discriminates between parallel and antiparallel/mixed ordered aggregates. 
Figure 12: MTT assay after $48 \mathrm{hrs}$ of treatment of SHSY5Y cell cultures with different 17-29 h-amylin preparations $(25 \mu \mathrm{M})$. Data are means \pm S.D. $(\mathrm{n}=4)$ of 3 independent experiments, and expressed as percent vs. control. Statistical analysis was performed by one way ANOVA, all Pairwise Multiple Comparison Procedures (Holm-Sidak method): significant comparisons are indicated by horizontal curly brackets.

Figure 13: MTT assay after $48 \mathrm{hrs}$ of treatment of insulinoma (RIN-m) cell cultures with different h-amylin 17-29 preparations $(25 \mu \mathrm{M})$. Data are means \pm S.D. $(\mathrm{n}=5)$ of 2 independent experiments, and expressed as percent vs. control. Statistical analysis was performed by one way ANOVA, all Pairwise Multiple Comparison Procedures (Holm-Sidak method): significant comparisons are indicated by horizontal curly brackets.

Figure 14: MTT assay after $48 \mathrm{hrs}$ of treatment of neuroblastoma (SHSY5Y) cell cultures with different h-amylin 1-37 preparations $(20 \mu \mathrm{M})$. Data are means \pm S.D. $(n=5)$ of 2 independent experiments, and expressed as percent vs. control. Statistical analysis was performed by one way ANOVA, all Pairwise Multiple Comparison Procedures (Holm-Sidak method): significant comparisons are indicated by horizontal curly brackets.

Figure 15: MTT assay after $48 \mathrm{hrs}$ of treatment of insulinoma cell cultures with different h-amylin 1-37 preparations $(20 \mu \mathrm{M})$. Data are means \pm S.D. $(n=4)$ of 3 independent experiments, and expressed as percent vs. control. Statistical analysis was performed by one way ANOVA, all Pairwise Multiple Comparison Procedures (Holm-Sidak method): significant comparisons are indicated by horizontal curly

brackets. 


\section{References}

1 M. L. Giuffrida, F. Caraci, B. Pignataro, S. Cataldo, P. De Bona, V. Bruno, G. Molinaro, G. Pappalardo, A. Messina, A. Palmigiano, D. Garozzo, F. Nicoletti, E. Rizzarelli and A. Copani, Betaamyloid monomers are neuroprotective, J. Neurosci. 2009, 29, 10582-10587.

${ }^{2}$ A. S. De Toma, S. Salamekh, A. Ramamoorthy and M. H. Lim, Misfolded proteins in Alzheimer's disease and type II diabetes, Chem. Soc. Rev. 2012, 41, 608-621.

${ }^{3}$ L. K. Phillips and M. Horowitz, Amylin, Curr. Opin. Endocrinol. Diab. 2006, 13, 191-198.

${ }^{4}$ P. Cao, A. Abedini and D. P. Raleigh, Aggregation of islet amyloid polypeptide: from physical chemistry to cell biology, Curr. Opin. Struct. Biol. 2013, 23, 82-89.

${ }^{5}$ P. Westermark, A. Andersson and G. T. Westermark, Islet amyloid polypeptide, islet amyloid and diabetes mellitus, Physiol. Rev. 2011, 91, 795-826.

${ }^{6}$ P. Westermark, U. Engström, K. H. Johnson, G. T. Westermark and C. Betsholtz, Islet amyloid polypeptide: pinpointing amino acid residues linked to amyloid fibril formation, Proc. Natl. Acad. Sci. U.S.A. 1990, 87, 5036-5040.

${ }^{7}$ B. Konarkowska, J. F. Aitken, J. Kistler, S. Zhang and G. J. Cooper, The aggregation potential of human amylin determines its cytotoxicity towards islet beta-cells, FEBS J. 2006, 273, 3614-3624.

${ }^{8}$ N. F. Dupuis, C. Wu, J.-E. Shea and M. T. Bowers, The amyloid formation mechanism in human IAPP: dimers have $\beta$-strand monomer-monomer interfaces, J. Am. Chem. Soc. 2011, 133, 7240-7243.

${ }^{9}$ V. L. Sedman, S. Allen, W. C. Chan, M. C. Davies, C. J. Roberts, S. J. Tendler and P. M. Williams, Atomic force microscopy study of human amylin (20-29) fibrils, Protein Peptide Lett. 2005, 12, 7983. 
${ }^{10}$ G. Pappalardo, D. Milardi, A. Magrì, F. Attanasio, G. Impellizzeri, C. La Rosa, D. Grasso and E. Rizzarelli, Environmental factors differently affect human and rat IAPP: conformational preferences and membrane interactions of IAPP17-29 peptide derivatives, Chemistry 2007, 13, 10204-10215.

${ }^{11}$ A. T. Alexandrescu, Amyloid accomplices and enforcers, Protein Sci. 2005, 14, 1-12.

${ }^{12}$ S. Jha, S. M. Patil, J. Gibson, C. E. Nelson, N. N. Alder and A. T. Alexandrescu, Mechanism of amylin fibrillization enhancement by heparin. J. Biol. Chem. 2011, 286, 22894-22904.

${ }^{13}$ S. M. Mirhashemi and M. H. Aarabi, Effect of two herbal polyphenol compounds on human amylin amyloid formation and destabilization, J. Med. Plants Res. 2012, 6, 3207-3212.

${ }^{14}$ V. Chien, J. F. Aitken, S. Zhang, C. M. Buchanan, A. Hickey, T. Brittain, G. J. S. Cooper and K. M. Loomes, The chaperone proteins HSP70, HSP40/DnaJ and GRP78/BiP suppress misfolding and formation of $\beta$-sheet-containing aggregates by human amylin: a potential role for defective chaperone biology in type 2 diabetes, Biochem. J. 2010, 432, 113-121.

${ }^{15}$ B. Cheng, H. Gong, X. Li, Y. Sun, X. Zhang, H. Chen, X. Liu, L. Zheng and K. Huang, Silibinin inhibits the toxic aggregation of human islet amyloid polypeptide, Biochem. Bioph. Res. Co. 2012, 419, 495-499.

${ }^{16}$ L. H. Tu and D. P. Raleigh, Role of aromatic interactions in amyloid formation by islet amyloid polypeptide, Biochemistry 2013, 52, 333-342.

${ }^{17}$ M. Kamihira-Ishijima, H. Nakazawa, A. Kira, A. Naito and T. Nakayama, Inhibitory mechanism of pancreatic amyloid fibril formation: formation of the complex between tea catechins and the fragment of residues 22-27, Biochemistry 2012, 51, 10167-10174.

${ }^{18}$ P. Cao and D. P. Raleigh, Analysis of the inhibition and remodeling of islet amyloid polypeptide amyloid fibers by flavanols, Biochemistry 2012, 51, 2670-2683. 
${ }^{19}$ B. Cheng, X. Liu, H. Gong, L. Huang, H. Chen, X. Zhang, C. Li, M. Yang, B. Ma, L. Jiao, L. Zheng and K. Huang, Coffee components inhibit amyloid formation of human islet amyloid polypeptide in vitro: possible link between coffee consumption and diabetes mellitus, J. Agric. Food Chem. 2011, 59, $13147-13155$.

${ }^{20}$ M. Pannuzzo, A. Raudino, D. Milardi, C. La Rosa and M. Karttunen, $\alpha$-helical structures drive early stages of self-assembly of amyloidogenic amyloid polypeptide aggregate formation in membranes, Scientific Reports 2013, 3, 2781.

${ }^{21}$ M. F. M. Sciacca, D. Milardi, G. M. L. Messina, G. Marletta, J. R. Brender, A. Ramamoorthy and C. La Rosa, Cations as switches of amyloid-mediated membrane disruption mechanisms: calcium and IAPP, Biophys. J. 2013, 104, 173-184.

22 J. R. Brender, K. Hartman, R. P. Nanga, N. Popovych, R. de la Salud Bea, S. Vivekanandan, E. N. Marsh and A. Ramamoorthy, Role of zinc in human islet amyloid polypeptide aggregation, J. Am. Chem. Soc. 2010, 132, 8973-8983.

${ }^{23}$ B. Ward, K. Walker and C. Exley, Copper(II) inhibits the formation of amylin amyloid in vitro, $J$. Inorg. Biochem. 2008, 102, 371-375.

${ }^{24}$ Y.P. Yu, P. Lei, J. Hu, W. H. Wu, Y. F. Zhao and Y. M. Li, Copper-induced cytotoxicity: reactive oxygen species or islet amyloid polypeptide oligomer formation, Chem. Comm. 2010, 46, 6909-6911.

${ }^{25}$ C. Kállay, A. Dávid, S. Timári, E. M. Nagy, D. Sanna, E. Garribba, G. Micera, P. De Bona, G. Pappalardo, E. Rizzarelli and I. Sóvágó, Copper(II) complexes of rat amylin fragments, Dalton Trans. 2011, 40, 9711-9721.

${ }^{26}$ K. Lemaire, M. A. Ravier, A. Schraenen, J. W. M. Creemers, R. Van de Plas, M. Granvik, L. Van Lommel, E. Waelkens, F. Chimienti, G. A. Rutter, P. Gilon, P. A. in't Veld and F. C. Schuit, Insulin 
crystallization depends on zinc transporter ZnT8 expression, but is not required for normal glucose homeostasis in mice, Proc. Natl. Acad. Sci. U.S.A. 2009, 106, 14872-14877.

${ }^{27}$ A. Masad, L. Hayes, B. J. Tabner, S. Turnbull, L. J. Cooper, N. J. Fullwood, M. J. German, F. Kametani, O.M. El-Agnaf and D. Allsop, Copper-mediated formation of hydrogen peroxide from the amylin peptide: A novel mechanism for degeneration of islet cells in type-2 diabetes mellitus?, FEBS Lett. 2007, 581, 3489-3493.

${ }^{28}$ C. E. E. House, T. Patel, L. Wu and P. E. Fraser, Human pro-islet amyloid polypeptide (ProIAPP148) forms amyloid fibrils and amyloid spherulites in vitro, J. Inorg. Biochem. 2010, 104, 1125-1129.

${ }^{29}$ F. Bellia and G. Grasso, The role of copper(II) and zinc(II) in the degradation of human and murine IAPP by insulin-degrading enzyme, J. Mass Spectrom. 2014, 49, 274-279.

${ }^{30}$ M. J. Kim and H. T. Kim, Investigation of the copper binding site on the human islet amyloid polypeptide hormone, Eur. J. Mass Spectrom. 2012, 18, 51-58.

${ }^{31}$ R. G. Bennett, W. C. Duckworth and F. G. Hamel, Degradation of amylin by insulin-degrading enzyme, J. Biol Chem. 2000, 275, 36621-36625.

${ }^{32}$ H. Guan, K. M. Chow, R. Shah, C. J. Rhodes and L. B. Hersh, Degradation of islet amyloid polypeptide by neprilysin, Diabetologia 2012, 55, 2989-2998.

${ }^{33}$ K. Aston-Mourney, S. Zraika, J. Udayasankar, S. L. Subramanian, P. S. Green, S. E. Kahn and R. L. Hull, Matrix metalloproteinase-9 reduces islet amyloid formation by degrading islet amyloid polypeptide, J. Biol. Chem. 2013, 288, 3553-3559.

${ }^{34}$ G. Grasso, F. Salomone, G. R. Tundo, G. Pappalardo, C. Ciaccio, G. Spoto, A. Pietropaolo, M. Coletta and E. Rizzarelli, Metal ions affect insulin-degrading enzyme activity, J. Inorg. Biochem. 2012, $117,351-358$. 
${ }^{35}$ G. Grasso, M. L. Giuffrida and E. Rizzarelli, Metal ions and proteolytic enzymes in Alzheimer's disease, Metallomics, 2012, 4, 937-949.

${ }^{36}$ G. Grasso, A. Pietropaolo, G. Spoto, G. Pappalardo, G. R. Tundo, C. Ciaccio, M. Coletta and E. Rizzarelli, Copper(I) and copper(II) inhibit $\mathrm{A} \beta$ peptides proteolysis by insulin-degrading enzyme differently: implications for metallostasis alteration in Alzheimer's disease, Chemistry, 2011, 17, $2752-$ 2762.

${ }^{37}$ L. A. Ralat, V. Kalas, Z. Z. Zheng, R. D. Goldman, T. R. Sosnick and W.-J. Tang, Ubiquitin is a novel substrate for human insulin-degrading enzyme, J. Mol. Biol. 2011, 406, 454-466.

${ }^{38}$ F. Bellia, A. Pietropaolo and G. Grasso, Formation of insulin fragments by insulin-degrading enzyme: the role of zinc(II) and cystine bridges, J. Mass Spectrom. 2013, 48, 135-140.

${ }^{39}$ G. Grasso, E. Rizzarelli and G. Spoto, How the binding and degrading capabilities of insulin degrading enzyme are affected by ubiquitin, BBA-Protein Proteom. 2008, 1784, 1122-1126.

${ }^{40}$ C. Ciaccio, G. F. Tundo, G. Grasso, G. Spoto, D. Marasco, M. Ruvo, M. Gioia, E. Rizzarelli and M. Coletta, Somatostatin: a novel substrate and a modulator of insulin degrading enzyme activity, J. Mol. Biol. 2009, 385, 1556-1567.

${ }^{41}$ Y. Shen, A. Joachimiak, M. R. Rosner and W.-J. Tang, Structures of human insulin-degrading enzyme reveal a new substrate recognition mechanism, Nature 2006, 443, 870-874.

${ }^{42}$ Q. Guo, M. Manolopoulou, Y. Bian, A. B. Schilling and W.-J. Tang, Molecular basis for the recognition and cleavages of IGF-II, TGF- $\alpha$, and amylin by human insulin-degrading enzyme, J. Mol. Biol., 2010, 395, 430-443.

${ }^{43}$ N. R. Williams, J. Rajput-Williams, J. A. West, S. V. Nigdikar, J. W. Foote and A. N. Howard, Plasma, granulocyte and mononuclear cell copper and zinc in patients with diabetes mellitus, Analyst 1995, 120, 887-890. 
${ }^{44}$ W. Fu, A. Patel and J.H. Jhamandas, Amylin receptor: a common pathophysiological target in Alzheimer's disease and diabetes mellitus, Front. Aging Neurosci. 2013, 42, 1-4.

${ }^{45}$ G. Grasso, E. Rizzarelli and G. Spoto, The proteolytic activity of insulin-degrading enzyme: a mass spectrometry study, J. Mass Spectrom. 2009, 44, 735-741.

${ }^{46}$ Y. P. Yu, P. Lei, J. Hu, W. H. Wu, Y. F. Zhao and Y. M. Li, Copper-induced cytotoxicity: reactive oxygen species or islet amyloid polypeptide oligomer formation, Chem. Commun. 2010, 46, 69096911.

${ }^{47}$ J. Madine, E. Jack, P. G. Stockley, S. E. Radford, L. C. Serpell and D. A. Middleton, Structural insights into the polymorphism of amyloid-like fibrils formed by region 20-29 of amylin revealed by solid-state NMR and x-ray fiber diffraction, J. Am. Chem. Soc. 2008, 130, 14990-15001. 